\title{
Lutte De Leadership Feminin Au Pouvoir Et (Re) Structuration De Rapports Sociaux De Sexe En Province Orientale
}

\author{
Bibiche Liliane Salumu Laumu Omeyaka \\ Chercheuse à la Faculté de Sciences Sociales, Administratives et Politiques \\ de l'Université de Kisangani (UNIKIS) et au Centre de Recherches \\ Politiques et Sociales d'Afrique Noire (CEREPSAN)/ RD Congo
}

Doi:10.19044/esj.2018.v14n35p84 URL:http://dx.doi.org/10.19044/esj.2018.v14n35p84

\begin{abstract}
This paper focuses on the subversive and performative gender dimension of the emergence of women politicians in post-Mobutu Oriental Province. The ambition of these women political leaders, in a bid to maintain their status as women's elite, derives its livelihood in the implementation of their ability. This capability creates social change through social deconstruction of gender roles in the society structural transformations known as neo "patriarchal" and also in the heterosexual relationship (man-woman) and homosexual relationship (women-women) known as "transformations interactionists". From this perspective, these elites are indeed agents of change for the welfare of women in their province. This way of acting was being made from them women's elites of conviction. The opposite, however, were also observed when some of them resigned themselves from being able to break the glass wall sheltering the inequalities between the sexes for others especially political purposes. They are selective in their commitment. In this case, it is women's elites of convention serving, most often times, as a gateway to politics. This entrepreneurship of women political leaders causes the political subsystem to change into neo-patriarchalism. It is through the narratives of life coupled interviews that we have managed to highlight the particularities of these women determined to forge a personality in a cultural, social, and political space that is very constraining for them.
\end{abstract}

Keywords: Women political leaders, ability, social mobility, power relations, social transformations, political participation

\section{Résumé}

Cet article aborde la dimension subversive et performative de genre de l'émergence des femmes leaders politiques en Province Orientale post- 
Mobutu. L'ambition de ces femmes leaders politiques de maintenir leur statut d'élite féminine tire sa subsistance dans la mise en œuvre de leur capabilité. Cette capabilité engendre des transformations sociales à travers une déconstruction sociale de rôle des sexes dans la société "transformations structurelles dites néo patriarcales» mais aussi dans le rapport hétérosexué (homme-femme) et le rapport intrasexué (femme-femme) «transformations interactionnistes ». Sous cette optique, ces élites sont effectivement des agents de changement pour le bien être des femmes dans leur province.

Cette manière d'agir et d'être fait d'elles des élites féminines de conviction mais le contraire est également observé lorsque certaines d'entre-elles se résignent à pouvoir briser le mur de verre abritant les inégalités entre les sexes pour d'autres fins notamment politiciennes. Elles sont sélectives dans leur engagement. Dans ce cas, ce sont des élites féminines de convention servant, le plus souvent, de passerelle aux politiques. Cet entreprenariat des femmes leaders politiques fait que le sous-système politique se modifie en néopatriarcalisme. C'est à travers les récits de vie accouplés des interviews que nous avons réussi à ressortir les particularités de ces femmes déterminées à se forger une personnalité dans un espace culturel, social et politique très contraignant pour elles.

Mots clés: femmes leaders politiques, capabilité, Rapport de pouvoir hommefemme, transformations sociales, participation politique.

\section{Introduction}

L'émergence des femmes leaders en politique est un enjeu de la gouvernance politique de toute société surtout celle résiliente qui tient à son équilibre, son harmonie et son développement. Mais, dans le même temps, il faut admettre que, autant que les hommes, les femmes proviennent de diverses origines géographiques, politiques, culturelles, linguistiques, religieuses, etc. qui les façonnent et qu'elles façonnent. Des origines qui tracent leur trajectoire, orientent leur perspectives, leur vision, leurs aspirations ; bref, leur être. Elles sont objet de leur propre représentation au-delà de ce que véhicule l'idéologie dominante «patriarcale» en Province Orientale, comme l'a souligné Fatou Sow (, 2009).

Nous avons ainsi tenu à passer en revue l'engagement des militantes au sein des partis politiques à travers lequel les femmes ont révolutionné l'espace politique congolais, en général et en Province Orientale, en particulier. Il s'agit, donc, de questionner leur trajectoire sociologique sur laquelle se sont engagées les congolaises de cette province, dans leur confirmation, leur autodétermination et leur autonomie dans l'espace social et politique. Dès lors, les témoignages auxquels nous avons recourus dans ce papier mettent en lumière, à la fois, une perception critique du rôle souvent attribué aux femmes 
et une valorisation des modèles traditionnels féminins, ainsi que de ceux proposés par la société « bourgeoise » ou « moderne » en pleine expansion.

Par ailleurs, la compréhension de l'émergence des femmes leaders en politique dans la Province Orientale nous amène à considérer les sexes hors de la nature, de l'impensé, du présocial, et de les projeter dans l'histoire, dans le social et dans la politique. En effet, tout un ensemble de travaux dont ceux de Monique Wittig ( 2001) et Judith Butler ( 2005) sont les plus significatifs, ont mis en lumière le fait que le système de genre est constitué par un ensemble de prescriptions normatives au sein desquelles l'enchaînement linéaire entre «l'être femme, l'être féminine et l'être hétérosexuée» occupe une place centrale ${ }^{3}$.

Toute entrave à cette continuité comporte des coûts sociaux et politiques plus ou moins lourds à porter selon les contextes historiques et politiques. Ainsi, l'appartenance de sexe a une importance centrale dans l'enchaînement normatif de ces trois éléments. De ce fait, nous sommes dans une double posture dénommée l'anti-essentialisme-constructivisme. Le statut de ces femmes leaders constitue la victoire de l'ancrage entre les principes générateurs communs basés sur la valence différentielle de sexes et ceux axés sur les choix individuels ou de préférences individuelles. Cette victoire nous pousse à croire que les femmes leaders politiques n'ont pas toutes intériorisées le référencement de l'enchainement normatif précité de la même manière. Il y a, certes des femmes qui vivent leur féminité en crise en faveur d'une masculinité, et d'autres vivent le contraire.

Le processus analysé nous montre, non seulement la diversité discursive des sujets qui s'articulent, mais également les processus de déconstruction et reconstruction des identités de celles qui ont émergé en politique. Le recours au corpus idéologique s'avère capital pour rendre compte des ensembles cohérents de représentations mentales relatifs à l'organisation sociale et politique. Cependant, une définition compréhensive doit prendre en considération leur aspect dynamique, c'est-à-dire leur capacité à influencer les pratiques sociales à travers un processus de (re) construction du réel qu'elles induisent. C'est pourquoi nous désignons, sous le mot idéologie, les systèmes de représentations qui fonctionnent doublement à la croyance culturelle et à la viabilité institutionnelle.

L'accès aux données est conditionné par l'usage des techniques entendues, ici, comme des moyens précis pour atteindre un résultat partiel, à un niveau et à un moment précis de la recherche. Cette atteinte de résultat est directe et relève du concret, du fait observé, de l'étape pratique et limitée. Ce sont des outils momentanés, conjoncturels et limités dans le processus de

${ }^{3}$ Le même schéma linéaire s'applique bien entendu aussi aux hommes : homme, masculin, hétérosexuel. 
recherche : sondage, interview semi-structuré, technique documentaire, organisation des séminaires de partage d'expérience et de renforcement de capacités des femmes politiques, l'observation participante en qualité d'actrice de la société civile accompagnant les femmes engagées en politique ont été mobilisés à cet effet.

La taille de notre échantillon mixte était de 50 sujets. Le nombre de femmes députées provinciales (sept) et ministres (deux) était connu, mais les statistiques des femmes militantes politiques non détentrice du pouvoir ou présidentes de ligues des femmes de partis politiques n'étaient pas disponibles lors de notre recherche. Nous avons pu échanger avec 41 militantes politiques dont la plupart était de candidates aux élections de 2006 et 2011. Le recours à la technique documentaire était recommandé pour contourner cette difficulté. Le choix raisonné se justifie du fait d'interviewer certaines personnes ressources possédant une idée sur les figures de réussite politique et sociale, l'exercice du pouvoir politique en Province Orientale se rapportant aux dynamiques sociales et politiques relatives aux questions genre.

Nous avons fait recours au codage (Bardin L., 2016) comme technique de traitement des données tirées de témoignages des femmes leaders politiques interviewées, nous avons utilisé l'analyse qualitative appuyé par l'analyse structurale (mises en sens, mises en pratique des normes socialement construites) et l'analyse de discours prononcés par ces femmes par rapport à la promotion du genre en Province Orientale.

Cette réflexion s'inscrit dans une sociologie des « actrices » nommées femmes leaders politiques en Province Orientale ${ }^{4}$ à travers les différentes fonctions politiques exercées par les femmes politiques dans cette province. Ces femmes émergentes sont considérées comme des élites féminines au sens de Wilfredo Pareto (Grawitz M. et Leca J., 1979, p. 275), à savoir « les gens qui ont à un degré remarquable de qualités d'intelligence, de caractère, d'adresse, de capacité de tout genre ${ }^{5} »$.

Cette étude couvre la période allant de 1997 à 2015 en Province Orientale $^{6}$. Le choix de l'année 1997 se justifie par le passage d'une transition politique pacifique à celle d'une politique violente (militarisée), L'année 2015

${ }^{4}$ Cette étude est la continuité des travaux de Jean Omasombo sur les biographies des élites politiques congolaises de la transition et celles de la troisième république (Omasombo J. et Kennes E., 2006.) (Omasombo J., 2009)

${ }^{5}$ Ces femmes sont qualifiées de leaders par le fait de transcender les barrières socioculturels, stéréotypes véhiculés autours de la femme ayant une responsabilité publique. Nous reconnaissons avec Vilfredo Pareto que la plupart des actions sociales sont non logiques, suscitées par un état d'esprit non rationnel; ces actions sont en fait commandées en fait par les sentiments et les impulsions. Mais la rationalité de ces femmes leaders se fonde sur la conquête et l'exercice du pouvoir.

${ }^{6}$ La Province Orientale est subdivisée en quatre districts : Bas-Uélé, Haut-Uélé, Tshopo et Ituri 
se justifie par le fait du démembrement de la Province Orientale en quatre provinces autrefois districts (Bas-Uélé, Haut-Uélé, Ituri et Tshopo).

La compréhension de leadership féminin en politique requiert un regard sur la participation des femmes de la Province Orientale à travers la gestion des institutions publiques (I). De ce regard découlera les effets de la participation politiques des femmes sur les femmes au pouvoir et sur les rapports sociaux de sexes (II) et discussions de résultats (III).

\section{Participation des femmes de la Province Orientale dans la gestion des institutions publiques}

Nous décrivons les différentes rétributions de femmes leaders politiques à travers les fonctions exercées par les femmes dans les différentes institutions politiques et administratives en RDC : Assemblées (nationale et provinciale) et les différentes nominations des femmes aux Gouvernements et à d'autres fonctions politiques.

\section{Assemblées (nationale et provinciale)}

Cette partie retrace les différentes manières de désignation (accession aux instances du pouvoir) des membres de l'organe législatif à savoir : nomination, cooptation et élection.

A. Nomination des députés de l'Assemblée Constituante et LégislativeParlement de Transition (ACL-PT ) sous le régime de Laurent Désiré Kabila de 2000-2003

La formation de l'Assemblée Constituante et Législative- Parlement de Transition (ACL-PT, en sigle) a été effective à travers la promulgation du décret-loi constitutionnel $n^{\circ}$ 096/2000 du 21 juillet 2000 portant Organisation et exercice du pouvoir en RDC. Cette Assemblée parlementaire mise en place siégeait à Lubumbashi, dans la Province du Katanga. Elle était constituée des 300 membres appelés députés dont les membres du Bureau ont été nommés par le Président de la République. La proposition des noms de candidats députés a été faite par la commission de sélection de 30 membres chargée d'examiner les dossiers de candidatures. Les dossiers de candidatures étaient déposés au Secrétariat du Ministère de l'Intérieur. Cette commission instituée par le Chef de l'Etat, internée au palais de la nation avait deux semaines pour rendre son rapport de traitement de dossiers. La particularité de cette Assemblée parlementaire réside au niveau de la présidence attribuée à la femme, l'Ingénieur Philomène Omatuku. L'ACL-PT a été la première institution politique congolaise à être présidée par une femme.

Par ailleurs, cette Assemblée nationale comptait 270 hommes et 30 femmes députés nommés. La province orientale avait vingt-un députés dont quinze hommes et six femmes. Marceline Daruwezi Apendeki, Marie Moke 
Mambango, Marguerite Niki Imayonda, Scolastique Atadrazura, Alphonsine Asibu, et Eugenie Agoyo étaient ces femmes députées. Certaines d'entre-elles vont se maintenir en exerçant des fonctions politiques tant au niveau national que provincial comme on peut s'en rendre compte dans les lignes suivantes.

B. Cooptation des membres de l'Assemblée nationale sous la transition 1+4

Nous considérons la cooptation comme le mécanisme par lequel la désignation mutuelle des acteurs est faite sur la base d'un consensus des membres du groupe concerné. Le recours à ce mécanisme s'est observé lors de la transition 1+4 et de suppléance en cas de remplacement des députés décédés ou promus à d'autres postes.

La signature de l'Accord global et inclusif issu du Dialogue Inter Congolais (DIC, en sigle) en juin 2003, a marqué l'entrée dans une période de transition non violente au cours de laquelle des institutions nouvelles ${ }^{7}$ ont été mises en place afin d'entamer le processus de reconstruction post conflit. Ces institutions devaient permettre de gouverner le pays jusqu'à la tenue d'élections en 2006.

L'institutionnalisation de la transition $1+4^{8}$ est une de résolution des travaux de DIC. Le fonctionnement des institutions de la transition était basé sur les principes de la consensualité, de l'inclusivité et de la non-conflictualité. La répartition des responsabilités au sein des institutions de la transition et à tous les niveaux de l'Etat était basée sur le principe de l'inclusivité et du partage équitable entre les composantes et entités au DIC. Ces composantes et entités étaient constituées des acteurs suivants : Le Gouvernement de la République Démocratique du Congo, les mouvements rebelles [le Rassemblement Congolais pour la Démocratie (RCD), le Mouvement de Libération du Congo (MLC), le Rassemblement Congolais pour la Démocratie/Mouvement de Libération (RCD/ML), le Rassemblement Congolais pour la Démocratie/National (RCD/N), les Maï-Maï;], l'Opposition politique et les Forces vives. Malgré les principes susmentionnés, la répartition des postes fut

\footnotetext{
${ }^{7}$ Ces nouvelles institutions renvoient aux institutions ci-après: le Président de la République, le Gouvernement, l'Assemblée nationale, le Sénat et les Cours et les tribunaux. En plus des Institutions ci-dessus, étaient créées les institutions d'appui à la démocratie suivantes : la Commission électorale indépendante, l'Observatoire national des droits de l'Homme, la Haute autorité des médias, la Commission vérité et réconciliation et la Commission de l'éthique et de la lutte contre la corruption.

${ }^{8}$ La transition $1+4$ désigne un mode exceptionnel dans lequel le pouvoir politique était géré par le président de la République (1) et quatre vice-présidents (4). Chaque Vice-Président était en charge d'une des quatre commissions gouvernementales suivantes: La commission politique : Composante RCD, la commission économique et financière : Composante MLC, la commission pour la reconstruction et le développement : Composante Gouvernement et la Commission sociale et culturelle : Composante Opposition politique.
} 
largement influencée par les rapports de force existant entre ces différents acteurs impliqués dans la transition $1+4$.

Le tableau 1 nous retrace certaines femmes provenant de la Province Orientale ayant été cooptées à l'Assemblée Nationale de la Transition 1+4.

Tableau 1 : Des femmes cooptées à l'Assemblée Nationale de la Transition 1+4

\begin{tabular}{|r|c|c|}
\hline $\mathbf{N}^{\circ}$ & Noms de la femme cooptée & Composantes \\
\hline $\mathbf{1}$ & Jeannine Aiwa Aroyo Yikoba (Haut-Uélé) & Mouvement de Libération du Congo \\
\hline $\mathbf{2}$ & Jeanne Ikombele Baelongandi (Tshopo) & Mouvement de Libération du Congo \\
\hline $\mathbf{3}$ & Mimy Mopunga Makende (Equateur) & $\begin{array}{c}\text { Rassemblement Congolais pour la } \\
\text { Démocratie/Goma }\end{array}$ \\
\hline $\mathbf{4}$ & Marie-Hélène Wawa (Bas-Uélé) & $\begin{array}{c}\text { Rassemblement Congolais pour la } \\
\text { Démocratie/Goma }\end{array}$ \\
\hline $\mathbf{5}$ & Pétronelle Vaweka Rutaya (Ituri) & Opposition politique non armée (quota : Ituri) \\
\hline $\mathbf{6}$ & Marguerite Niki Imayonda (Tshopo) & Gouvernement \\
\hline $\mathbf{7}$ & Lucie Kipele Aky Azua (Bas-Uélé) & Société civile \\
\hline $\mathbf{8}$ & Béatrice Lomeya Atilite, (Haut-Uélé) & Société civile \\
\hline $\mathbf{9}$ & Bernadette Mabosu Mbay-Pati (Haut-Uélé) & Société civile \\
\hline
\end{tabular}

Il ressort de ce tableau 1 que les composantes et entités au DIC avaient listé les femmes sur leur proposition de belligérantes (MLC et RCD/Goma) ont aligné deux femmes issues de la Province Orientale. Par contre, l'opposition et le gouvernement ont eu un seul siège chacun où ils ont aligné des femmes. D'une manière générale, il faut noter que cette assemblée traduisait un déséquilibre genre. Les femmes étaient moins représentées que les hommes. Cette tendance est également nationale où l'Assemblée Nationale comptait $86 \%$ d'hommes contre $14 \%$ des femmes mais le taux de représentativité des femmes est légèrement supérieur à celui de la Transition de Laurent-Désiré Kabila qui avait $10 \%$, plus signification par rapport aux Assemblées nationales de 2006 (8,4\%) (Odimba C., 2012, p. 39) et de 2011 $(9,4 \%)$

Bien avant la formation de l'Assemblée Nationale 1+4, les autorités rebelles du RCD avaient installé une Assemblée Provinciale en Province Orientale durant la période comprise entre 2001 et 2003. Cette assemblée comptait 46 membres cooptés dont trois femmes: mesdames Bernadette Furaha, Béatrice Uzele et Marie-Hélène Wawa. Ces femmes étaient majoritairement membres de la société civile. Ces 46 membres cooptés jouissaient de la qualité d'honorable membre de ladite Assemblée. 
A. Election des députes femmes en Province Orientale en 2006 et 2011 Malgré les contraintes majeures faisant obstacle à l'élection des femmes ${ }^{9}$, certaines arrivent à briser le mur de verre ou à franchir le cap pour devenir sénatrices, députées nationales ou provinciales selon le cas.

Tableau 2 : Les femmes élues à l'Assemblée Nationale et Provinciale de la Province

Orientale 2006

\begin{tabular}{|c|c|c|c|}
\hline \multirow[t]{2}{*}{$\mathbf{N}^{\circ}$} & \multirow[t]{2}{*}{ Prénoms et Noms } & \multicolumn{2}{|c|}{ Elections aux instances politiques et partis politiques } \\
\hline & & Assemblée Nationale & Assemblée Provinciale \\
\hline 1 & Anastasie Moleko(Tshopo) & 2006/PPRD & \\
\hline 2 & Angèle Tabu (Ituri) & 2006/MSR & \\
\hline 3 & Caroline Ziana (Bas-Uélé) & & 2006/CODECO \\
\hline 4 & Elysée Mago (Haut-Uélé) & & $2006 / \mathrm{CCU}$ \\
\hline 5 & Espérance Shukuru (Ituri) & & 2006/Camp de la Patrie \\
\hline 6 & Georgette Bukani (Bas-Uélé) & 2006/MLC & \\
\hline 7 & $\begin{array}{l}\text { Hosanna Imbelenga (Haut- } \\
\text { Uélé) }\end{array}$ & & 2006/MSR \\
\hline 8 & Jeanne Abakuba (Haut-Uélé) & & 2006/PPRD \\
\hline 9 & Josphine Tshausiku (Bas-Uélé) & & 2006/PPRD \\
\hline 10 & Lucie Basonea (Bas-Uélé) & & 2006/PPRD \\
\hline 11 & Lucie Kipele (Bas-Uélé) & 2006/ARC & \\
\hline 12 & Marie Moke (Tshopo) & 2006/PPRD & \\
\hline
\end{tabular}

Ce tableau montre qu'en 2006 il y a eu 12 femmes qui ont été élues députées dont cinq à la députation nationale et sept à celle provinciale. Tous

\footnotetext{
${ }^{9}$ Les contraintes sont de trois ordres : 1) Les contraintes socioculturelles : les propos véhiculés traduisent les constructions sociales (règles, coutumes et croyances religieuses) du genre en défaveur de la femme par exemple: «la politique n'est pas une affaire des femmes» «que les femmes s'occupent de la maison" «Que les femmes se taisent dans les assemblées (1 corinthiens $14: 34)$. 2) les contraintes politiques : Notons que La RDC a ratifié bon nombre des instruments juridiques en faveurs de la femme notamment (I) Pacte International relatif aux Droits Civils et Politiques (PIDCP) conclu à New York le 16 décembre 1966, (II) Convention sur l'Elimination de toutes les formes de Discriminations à l'égard de la Femme, (III) Résolutions du Conseil de Sécurité des Nations Unies 1322,1820 et 1888, (IV) Objectifs du Millénaire pour le Développement, (V) Déclaration solennelle sur l'égalité entre les hommes et les femmes en Afrique en 2004, (Déclaration solennelle sur l'égalité entre les hommes et les femmes en Afrique en 2004 ; Protocole à la Charte africaine des droits de l'Homme et des peuples relatifs aux droits de la femme en Afrique ; Le protocole d'accord de la Communauté pour le Développement Economique de l'Afrique Australe (SADC) sur le genre et le Développement du 11décembre 2007, (VI) Protocole à la Charte africaine des droits de l'Homme et des peuples relatifs aux droits de la femme en Afrique, (VII) Protocole d'accord de la Communauté pour le Développement Economique de l'Afrique Australe (SADC) sur le genre et le Développement du 11décembre 2007. Mais la mise en œuvre des instruments juridiques ratifiés enregistre un faible impact à cause de la faible volonté politique. Les politiques congolais s'emploient plus souvent à soigner l'image de l'Etat congolais auprès des partenaires techniques et financiers notamment en élaborant les politiques publiques y afférentes : «l'émancipation de la femme avec Joseph-Désiré Mobutu sous l'influence de la décennie de la femme, la promotion de la femme avec Laurent-Désiré Kabila, et la politique nationale Genre avec Joseph Kabila. A titre illustratif, l'article 13 de la loi électorale de 2011 (LOI N${ }^{\circ} 11 / 003$ DU 25 JUIN 2011 MODIFIANT LA LOI N 06/006 DU 09 MARS 2006) ne permet pas la pleine application de la constitution en permettant de facto les limites dans la représentation des femmes es électorales n'est pas un motif d'irrecevabilité de la liste. 3) Les contraintes économiques : Les femmes congolaises ont un accès aux ressources économiques et naturelles plus limité que les hommes. La pauvreté en RDC est majoritairement féminine, ce qui limite leur capacité d'implication dans la vie politique.
} 
les quatre districts ont eu des femmes députées. Le District de Bas-Uélé a eu deux députés femmes à l'Assemblée Nationale et trois députés femmes à l'Assemblée Provinciale. Le District du Haut-Uélé n'avait eu que trois députés femmes à l'Assemblée Provinciale alors que l'Ituri avait une députée nationale et une autre provinciale. Quant au District de la Tshopo, il y a eu deux députés femmes à l'Assemblée Nationale. Mais la circonscription électorale Ville de Kisangani, il n'y a pas eu d'élection des femmes qu'il s'agisse de la députation Nationale que provinciale.

En ce qui concerne la configuration des partis politiques, ce sont ceux de 1'Alliance pour la Majorité Présidentielle (AMP) qui avait plus des députés femmes aux Assemblées. D'abord le PPRD avec deux députés à l'Assemblée Nationale et trois à l'Assemblée Provinciale. Les autres partis dont l'ARC avait une députée nationale; le MSR deux députés dont une nationale et une autre Provinciale. Le Camp de la Patrie avait une députée provinciale ; la CCU et la CODECO avait une députée Provinciale chacune. Du côté de l'opposition, le MLC avait une députée nationale.

Tableau 3. Les femmes élues à l'Assemblée Nationale en $2011^{10}$

\begin{tabular}{|c|c|c|}
\hline $\mathbf{N}^{\circ}$ & Prénoms et Noms & Assemblée Nationale \\
\hline 1 & Anastatie Moleko (Tshopo) & 2011/PPRD \\
\hline 2 & Angèle Tabu (Ituri) & 2011/MSR \\
\hline 3 & Béatrice Lomeya (Haut-Uélé) & 2011/PCD \\
\hline 4 & Espérance Shukuru (Ituri) & 2011/AHUDE \\
\hline 5 & Eve Bazaiba (Tshopo) & 2011/MLC \\
\hline 6 & Geneviève Inagosi (Haut-Uélé) & 2011/NAD \\
\hline 7 & Jeanne Ayomo $^{11}$ (Tshopo) & 2011/PPRD \\
\hline 8 & Lucie Kipele(Bas-Uélé) & 2011/Forces du Renouveau \\
\hline 9 & Marcelline Daruwezi (Tshopo) & $2011 / \mathrm{FC}$ \\
\hline
\end{tabular}

Ce tableau illustre qu'en 2011, le nombre de députés femmes a cru. Il y a eu au total neuf députés femmes dans les différents districts. Le District de la Tshopo est venu en premier lieu avec quatre députées femmes, dont trois de la Majorité Présidentielle (PPRD et FC) et une de 1'opposition (MLC). Le Haut-Uélé et l'Ituri avaient chacun deux députées toutes de la Majorité Présidentielle à savoir pour le Haut-Uélé : Béatrice Lomeya du PCD et

${ }^{10}$ Nous ne retenons ici la seule instance du fait que durant le processus électoral de 2011, il n'y a pas eu organisation des élections provinciales qui devraient se passer précisément en 2012. C'est la même Assemblée provinciale qui a continué à prester jusqu'en 2018.

${ }^{11}$ Madame Jeanne Ayomo était surnommée la benjamine des Commissaires du Peuple de la $4^{\text {ème }}$ législature de la deuxième République. Elle s'est présentée aux élections législatives de 1982 dans la circonscription électorale de la Tshopo, précisément le territoire d'Opala (HautZaïre). Elue avec 15.952 voix, elle s'est inscrite au Palais de la Nation dans la commission politique, administrative et judiciaire, dans la sous-commission de l'information. Elle faisait partie du groupe parlementaire de l'Union Interparlementaire au Zaïre (RDC). 
Geneviève Inagosi de la NAD. Le Bas-Uélé n'avait qu'une députée des Forces du Renouveau. La circonscription Ville de Kisangani n'a pas eu de députée Nationale femme aussi en 2016. Dans cette même logique, nous pouvons également aborder le cas de suppléance. Nos recherches ont montré que certaines femmes ont vu leur mandat parlementaire validé suite aux circonstances telles que la mort ou la promotion de leur titulaire. Le tableau ci-dessous nous présente la situation des femmes cooptées par suppléance à l'Assemblée Provinciale.

Tableau 4 : Des femmes cooptées par suppléance à l'Assemblée Provinciale en fonction des partis politiques

\begin{tabular}{|c|c|c|c|}
\hline $\begin{array}{c}\text { District de } \\
\text { provenanc } \\
\text { e }\end{array}$ & $\begin{array}{c}\text { Noms de la femme } \\
\text { cooptée }\end{array}$ & Député titulaire et Parti politique & Circonstance \\
\hline Bas-Uélé & Nasimo Sasa & Caroline Ziana Bolindani, CODECO & Décédée en 2011 \\
\hline Bas-Uélé & Godelive Anina Makwa & Jean Batamane Mosito, CDC & Décédé en 2011 \\
\hline Bas-Uélé & Aisha Kwanza & Dieudonné Basiliga Asembo, MLC & Décédée en 2011 \\
\hline Ituri & $\begin{array}{c}\text { Marie-Jeanne Dz'va } \\
\text { Kove }\end{array}$ & Jean Mbitso, PPRD & Nommé Ministre \\
\hline
\end{tabular}

Source : Notre propre enquête novembre 2014

Ce tableau 4 démontre que, dans certains districts notamment le Bas-Uélé et l'Ituti, les candidats avaient aligné les femmes comme leurs premières suppléantes, ce qui a favorisé leur émergence à l'hémicycle provincial. Mais dans le District de la Tshopo, aucun candidat n'a aligné la femme comme première suppléante. Donc, aucune femme n'a été élue ni coopté députée. Malgré cette stratégie d'alignement des femmes en position de suppléance utile, le taux des femmes est resté faible, soit huit sur les 96 députés provinciaux.

\section{Nomination des femmes de la Province Orientale aux Gouvernements et à d'autres fonctions politiques}

Sous la rébellion, certaines femmes ont collaboré avec les autorités du mouvement rebelle RCD. Cette collaboration a permis l'ascension des femmes sur la scène politique. Leur rétribution était sanctionnée par leur nomination à certains postes politiques. A titre d'exemple, Madame Adel Lotshove Mugisa était nommée Vice-gouverneur et Monsieur Bene Kabala Lothia, Gouverneur de la Province Orientale (du 26/09/1998 au 06/12/1998). Pour rappel, Madame Adel Lotshove Mugisa était militante très engagée dans la société civile. Elle était membre de l'ONG les Amis de Nelson Mandela pour les Droits Humains où elle assumait la fonction de la coordinatrice de la commission femme et enfant.

Par ailleurs, certaines femmes ont été promues ministres nationales ou provinciales et à d'autres fonctions politiques à cause de leur engagement sur la scène politique. 
Tableau 5: Quelques femmes promues à la territoriale en Province Orientale de 1998 à 2015

\begin{tabular}{|c|c|c|}
\hline $\mathbf{N}^{\circ}$ & $\begin{array}{c}\text { Noms et Post-noms et District } \\
\text { d'origine }\end{array}$ & Différentes fonctions \\
\hline \multicolumn{3}{|c|}{ A. Différentes fonctions à la territoriale de la Province Orientale } \\
\hline 1 & Jeanne Schuwa (Tshopo) & Bourgmestre de la commune Tshopo, 2007 \\
\hline 2 & Godelive Mosunga (Tshopo) & Bourgmestre de la Commune Kisangani, 2009 \\
\hline 3 & Marie-Thérèse Benda (Bas-Uélé) & Bourgmestre de la commune Tshopo, 2003 \\
\hline 4 & Marie Bhamoko Deuda (Haut-Uélé) & $\begin{array}{l}\text { Bourgmestre adjointe de la Commune Mangobo } \\
2012 \text { puis Lubunga } 2014 \\
\end{array}$ \\
\hline 5 & Marie Mimi Saile Selenge (Tshopo) & Bourgmestre de la Commune Lubunga 2005 \\
\hline 6 & Marie Senenge Sakina (Tshopo) & $\begin{array}{l}\text { Bourgmestre Adjoint de la Commune Kisangani, } \\
2000\end{array}$ \\
\hline 7 & Béatrice Uzele (Ituri) & $\begin{array}{l}\text { Bourgmestre Adjoint de la Commune Kisangani } \\
\qquad 2000\end{array}$ \\
\hline 8 & $\begin{array}{l}\text { Jeanne Abakuba Anyakimapa (Haut- } \\
\text { Uélé) }\end{array}$ & $\begin{array}{c}\text { Bourgmestre adjointe chargée des finances de la } \\
\text { commune de Mambaya à Isiro sous RCD/KML de } \\
\text { MBUSA } 2001\end{array}$ \\
\hline 9 & Sophie Kenda (Haut-Uélé) & Chef de cité Adjointe à Isiro 2013 \\
\hline 10 & Thérèse Sege (Bas-Uélé) & Chef de cité Adjointe à Buta \\
\hline 11 & Marie Avongi Sandrio Yele (Ituri) & Chef de secteur Ndo-Kebo à Alungba \\
\hline 12 & Clémentine Binti Darubu (Tshopo) & $\begin{array}{l}\text { Administratrice du Territoire d'Ubundu sous RCD, } \\
2000\end{array}$ \\
\hline 13 & Elisabeth Sandja Bokula (Tshopo) & $\begin{array}{l}\text { Administratrice du Territoire d'Isangi sous RCD, } \\
2000\end{array}$ \\
\hline 14 & $\begin{array}{c}\text { Lucie Kipele Aki Aky Azua (Bas- } \\
\text { Uélé) }\end{array}$ & $\begin{array}{l}\text { Administratrice du territoire assistante à Bili } \\
\text { (Territoire de Bondo) de 2002-2003, }\end{array}$ \\
\hline 15 & Celestine Ö̈li Lotimba (Tshopo) & Administratrice de Territoire de Basoko, 2008 à 2012 \\
\hline 16 & Petronelle Vaweka (Ituri) & Commissaire du District de l'Ituri, 2004 \\
\hline 17 & Godelive Semeli (Tshopo) & $\begin{array}{l}\text { Commissaire du District Assistante de la Tshopo, } \\
2008\end{array}$ \\
\hline \multicolumn{3}{|c|}{ B. Femmes de la Province Orientale ministres } \\
\hline 1 & Cécile Leteta Kumisa (Bas-Uélé) & Vice-ministre de la Justice sous Mozito III, 2011 \\
\hline 2 & Anastasie Moleko Moliwa (Tshopo) & $\begin{array}{l}\text { Ministre des affaires Sociales et Famille juin 1998- } \\
\text { septembre } 2000 \\
\text { Ministre du travail et Prévoyance sociale, ministre de } \\
\text { la Santé, septembre 2000-avril } 2001\end{array}$ \\
\hline 3 & Marguerite Niki Imayonda (Tshopo) & $\begin{array}{c}\text { Ministre provinciale de finances, économie et } \\
\text { portefeuille sous Autshai, } 2007 \\
\text { Ministre provinciale du Travail, Tourisme, Culture et } \\
\text { Arts sous Austhai, } 2008 \\
\text { Ministre provinciale de l'Enseignement Primaire et } \\
\text { Secondaire, Arts et Reforme culturelle sous } \\
\text { Bamanisa, 2012 } \\
\end{array}$ \\
\hline 4 & Angele Uvon (Ituri) & $\begin{array}{l}\text { Ministre provinciale de la Santé Publique, Affaires } \\
\text { Sociales,Genre et Humanitaires sous Austhai, } 2007\end{array}$ \\
\hline 5 & Jeanne Shuwa Bususu (Tshopo) & $\begin{array}{c}\text { Ministre provinciale de l'Agriculture et } \\
\text { Développement Rural sous Austhai, } 2009 \\
\text { Ministre provincial d'Energie, Développement } \\
\text { Rural,Tourisme, Culture et Art sous Austhai, } 2010\end{array}$ \\
\hline
\end{tabular}




\begin{tabular}{|r|c|c|}
\hline 6 & Jeanne Alasha (Ituri) & $\begin{array}{c}\text { Ministre provinciale de la Santé Publique, Affaires } \\
\text { Sociales,Genre et Humanitaires sous Bamanisa 2012 }\end{array}$ \\
\hline 7 & Jeanine Aiwa (Haut-Uélé) & $\begin{array}{c}\text { Ministre provinciale de l'Information, Sport, Loisirs } \\
\text { et Tourisme sous Bamanisa, 2012 }\end{array}$ \\
\hline & C. Autres fonctions importantes \\
\hline 1 & Carole Agito Amela (Bas-Uélé) & $\begin{array}{c}\text { Administratrice-Directrice Générale Adjointe } \\
\text { (ADGA) de la Société Nationale d'Assurance } \\
\text { (SONAS), 2008 }\end{array}$ \\
\hline 2 & Bernadette Tokwaulu Aena (Tshopo) & $\begin{array}{c}\text { Administratrice-Directrice Générale Adjointe } \\
\text { (ADGA) de la Société Nationale de l'électricité } \\
\text { (SNEL), 2008 }\end{array}$ \\
\hline 3 & Carol Kabanga Koy (Tshopo) & $\begin{array}{c}\text { Questeur à la Commission Electorale Nationale } \\
\text { Indépendante }{ }^{12} 2006\end{array}$ \\
\hline 4 & Evelyne Maway (Haut-Uélé) & $\begin{array}{c}\text { Conseillère de la République, Membre du conseil } \\
\text { économique et social de la RDC, 2014 }{ }^{13} .\end{array}$ \\
\hline 5 & Scolastique Atadrazura (Ituri) & $\begin{array}{c}\text { Conseillère de la République, Membre du conseil } \\
\text { économique et social de la RDC, 2014. }\end{array}$ \\
\hline 6 & Petronelle Madebe (Bas-Uélé) & $\begin{array}{c}\text { Conseillère de la République, Membre du conseil } \\
\text { économique et social de la RDC, 2014 }\end{array}$ \\
\hline
\end{tabular}

Ce tableau montre que les femmes ont émergé politiquement dans la territoriale. En effet, il y a eu 17 femmes qui ont occupé des fonctions dans ce secteur allant de l'Administrateur du Territoire au Commissaire de district en passant par le chef de cité et chef de secteur. Ceci est le fruit de leur leadership. Un autre secteur où les femmes ont su se confirmer entant qu'élite et leader a été l'institution provinciale, à savoir le Gouvernement Provincial. Certaines femmes qui s'étaient confirmées en leadership dans la territoriale ont pu occuper des postes ministériels au niveau de l'Exécutif provincial. C'est le cas de Jeanne Shuwa. Les autres y ont été élevées du fait de leur militantisme durant les rebellions (élite de guerre) tel est le cas de Jeanne Abakuba Anyakimapa et d'autres encore du fait de leur aura dans la défense des droits humains dans les organes de la société civile.

Le troisième secteur où les femmes se sont plus retrouvées a été les Entreprises et services publics, notamment la SONAS, la SNEL et l'institution d'appui à la démocratie, à savoir la CEI/CENI.

${ }^{12}$ Mme Carol Kabanga Koy était la $1^{\text {ère }}$ Rapport-Adjoint de la Commission Electorale Indépendante (CEI), chargée de la Commission Spéciale des Affaires Juridiques et du Contentieux.

${ }^{13}$ Le Conseil Economique et Social (CES) de la République Démocratique du Congo (RDC), prévu par la Constitution du 18 février 2006 (art. 208 à 210), s'est matérialisé par la Loi organique No 13/027 du 30 octobre 2013, portant organisation et fonctionnement du Conseil Economique et Social. Un effectif de 68 membres a aussitôt été investi en septembre 2014 et, le 16 décembre 2014. Les membres du CES-RDC appelés «Conseillers de la République » ont un mandat de cinq ans renouvelable. 
En interprétant ce tableau5, nous comprenons que le degré de participation aux activités des partis politiques constitue, parmi tant d'autres facteurs de rétribution, l'engagement militant d'un individu. A ce sujet, Daniel Gaxie (1979) fait observer que les difficultés rencontrées et les efforts consacrés à la réalisation de l'objectif politique que l'individu sert ne doivent pas être nécessairement considérés négativement dans ce calcul. Il rejoint la thèse d'Albert Hirschman (1982) pour qui, "le bénéfice de l'action collective n'est pas la différence entre le résultat qu'espère le militant et l'effort fourni, mais la somme de ces deux grandeurs». Nous pouvons dire que la nomination de ces femmes constitue leur rétribution pour leur dévouement ou loyauté aux idéaux soit de leurs partis politiques et/ou soit de leur allégeances aux parrains politiques. Mais leur rétribution n'est pas pareille à celle des hommes. Les entretiens ont révélé que depuis la nuit du temps, plus d'hommes sont nommés ministres et élus députés que les femmes. C'est maintenant que les femmes sont éveillées et qu'elles sont plus ou moins outillées pour la conquête du pouvoir. Ce qui n'est pas aussi facile pour elles, avait reconnu une députée provinciale $^{14}$

\section{Les effets de la participation politiques des femmes sur les femmes au pouvoir et sur les rapports sociaux de sexe.}

\subsection{Sur les femmes au pouvoir}

Les trois modes de désignation susmentionnés ont démontré la rétribution politique. Cette rétribution politique vient contribuer tant soit peu à la consolidation du leadership féminin en Province Orientale. S'agissant du leadership, Frederick George Bailey (1988) distingue deux formes idéalestypiques : celui d'équipes «morales» et celui d'équipes « transactionnelle». Le premier s'appuie sur la possibilité, pour le leader, de distribuer des rétributions morales ou honorifiques, alors que le second repose sur des rétributions matérielles. Par ailleurs, dans la jouissance de leur rétribution ont enregistré bel et bien une variance de mobilité sociale observable:

Mobilité géographique: La plupart des députées élues sont issues de territoire. Jusqu'en 2015, elles résidaient dans la ville de Kisangani où se trouvait le siège de l'Assemblée Provinciale de la Province orientale. Certaines ont quitté Wamba, Buta, Isiro, Ango pour Kisangani qui était le siège des institutions provinciales ou Kinshasa. Madame Cécile Leteta avait quitté Kisangani, sa ville de résidence, pour Kinshasa, capitale des institutions nationales (siège du gouvernement national). Madame Marie Avongi est partie du secteur Ndo-Kebo à Alungba en Ituri pour la capitale Kinshasa

Mobilité ascendante: Madame Espérance Shukuru est partie de la députation provinciale à la députation nationale), ou descendante: Mme

\footnotetext{
${ }^{14}$ Entretien du 14 avril 2014 réalisé à Kisangani.
} 
Cécile Leteta est partie de la vice-ministre nationale de la Justice à la chargée de contentieux à la Direction Générale des Recettes de la Province Orientale.

Mobilité biographique : acquisition d'une vie publique différente de celle ayant précédé l'exercice d'un mandat politique au niveau soit provincial ou national (voir les tableaux sur la cooptation, la nomination et l'élection à l'assemblée nationale et à l'assemblée provinciale).

Mobilité individuelle: Ces femmes ont transcendé les barrières politiques et sociales dressées contre la femme. Ces femmes leaders ont su séduire leur environnement social et politique en vue de décrocher la confiance de leur structure politique.

La compréhension de ces deux dernières mobilités appelle les commentaires de Michel Foucault (1976, p. 208). Selon cet auteur , le point du départ de cette résistance est l'instance du sexe qu'il faut s'affranchir si, par un retournement tactique des divers mécanismes de la sexualité, on veut faire valoir contre les prises du pouvoir, les corps, les plaisirs, les savoirs, dans leur multiplicité et leur possibilité de résistance. Contre le dispositif de sexualité, le point d'appui de la contre-attaque ne doit pas être le sexe-désir, mais les corps et les plaisirs. Les propos sur le tournant foucaldien est également repris dans le défaire le genre de Judith Butler pour exprimer une politique et une théorie de résistance contre les normes socialement construites en défaveurs de la femme.

Il s'observe que les femmes militantes politiques sont célibataires, mariées et veuves avec des enfants biologiques, adoptifs et/ou sous tuteurs. Elles sont détentrices de diplômes d'enseignement supérieurs et universitaire (Diplôme d'Etudes Supérieures, Licence et Graduat) et diplôme d'Etat. Par contre, les femmes n'ayant pas de diplôme d'Etat sont majoritaires contrairement à celles ayant de diplôme universitaire ou d'enseignement universitaire. Par ailleurs, les femmes députées par suppléances étaient pour la plupart des diplômées d'Etat (Baccalauréat), leur séjours politiques à Kisangani ont permis également leur inscription aux différentes institutions de l'Enseignement Supérieurs et Universitaires de la ville de Kisangani.

\subsection{Sur les rapports sociaux de sexes}

Avec ce profil diversifié des femmes leaders politiques, il est maintenant intéressant d'analyser de quelle manière la participation politique des femmes permet d'affirmer la capabilité des femmes leaders politique tant sur la gouvernance politique que dans les partis politiques.

\section{$1^{\circ}$ Sur la gouvernance politique}

Nos enquêtées ont avoué des difficultés auxquelles elles font face lors de l'exercice de leur mandat nominatif ou électif. Les entretiens avec les femmes détentrices du pouvoir (députées provinciales, ministres et 
bourgmestres) ont relevé qu'au sein du gouvernement, il y a un manque de volonté manifeste caractérisée par des discours mais peu d'actions avec moins d'effets faute de financement. Ce qui dénote un faible encouragement de la femme en politique ou l'inexistence des politiques publiques provinciales formelles, la persistance des pesanteurs socioculturelles rétrogrades. A titre d'exemple, la Province Orientale ne dispose pas d'un plan provincial de mise en ouvre de la résolution 1325 du conseil de Sécurité de Nations Unies. Ces propos de ces femmes viennent appuyer le constat du rapport national de l'audit genre des institutions publiques et privées de la RDC dans lequel le ministère du Genre, de la Famille et de l'Enfant dénonce que l'Assemblée provinciale et la Division Provinciale Genre de la Province Orientale n'ont pas des connaissances suffisantes des textes, des conventions et résolutions relatifs au Genre. Elles ont également une faible connaissance des problèmes et des débats actuels concernant le Genre. Au niveau de l'Assemblée Provinciale, il n'y a ni intégration de l'égalité homme-femme dans les objectifs et programmes d'action du ministère, ni ressources financières pour organiser les activités de promotion du Genre. En Province Orientale, il y a un vrai problème de perception et de compréhension du concept Genre et développement sur les activités spécialement axées sur la question de l'égalité homme-femme, c'est ainsi que même dans le budget, la question du Genre n'est pas prise en compte (Ministere du Genre, de la Famille et de l'Enfant, 2012, pp. 77, 85-86).

Cette situation favorise l'enracinement d'une forte disparité entre les sexes en défaveur de la militante politique. L'opinion publique considère que les femmes peuvent participer au champ politique, la seule restriction à leurs yeux étant celle qui exige que les femmes remplissent d'abord leur mission première d'éducation et de soin des enfants. Cette limite relative à la complémentarité des rôles reste donc une conviction fortement enracinée dans les conceptions qui, loin d'être exclusivement issues de la religion, sont partagées par toutes les sociétés fondées sur le système patriarcal (Alami Mchichi H., 2002). Les institutions politiques et les partis politiques constituent un lieu propice pour la pérennisation de ces faits.

A ce sujet, la question de différence des sexes s'impose. Ce qui nous a permis de mobiliser la valence différentielle des sexes de Françoise Héritier ( 1996) pour cerner certaines difficultés que rencontrent les femmes leaders dans l'exercice de leur militantisme politique.

Dans leurs rapports avec les hommes, les femmes interrogées ont affirmé que les hommes ont peur de l'engagement féminin, de l'inclusion des femmes en politique. Ils sous-estiment abusivement les aptitudes politiques des femmes à faire la politique et à reconnaître en tant que telles les compétences des femmes comme personnel politique féminin, le harcèlement sexuel pratiqué par certains hommes politiques. Ces faits évoqués montrent à quel 
point l'engagement des femmes n'est pas une chose facile. Judith Butler ( 2006) a eu à manifester ainsi sa vive inquiétude quant à la façon dont les luttes pour accéder à la reconnaissance du genre en général et pour obtenir un statut légitime sont susceptibles de contribuer à la perte de visibilité et à l'exclusion de certains entendez par là, les hommes.

De l'autre côté, le rapport entre les femmes se résument à la faible solidarité des femmes, à la mésentente entre les femmes, la jalousie, l'individualisme de certaines femmes émergentes, à la faible affirmation des femmes politiques dans la lutte pour l'autonomisation. Autrement dit, la plupart des femmes s'accrochent aux mots d'ordre de leur partis politiques quand bien même ceux-ci pêchent contre leur promotion, à la quête des facilités pratiquées par certaines femmes, le recours au clientélisme en lieu et place des efforts issus de dur label, faible aura auprès de la base, déficit de la vision politique. La faible solidarité féminine peut être considérée comme le produit d'une forte intériorisation de normes sociales en défaveur de la cohabitation et collaboration entre femmes. Ce que Pierre Bourdieu qualifie d'habitus qui dicte nos comportements de manière positive ou négative (Bourdieu P., 1980). Mais aussi, la question d'intérêt peut également diviser les femmes comme l'ont souligné Pat Heim \& Susan Murphy (2004) dans le monde du travail où les femmes entrent beaucoup plus souvent en conflit avec d'autres femmes qu'avec les hommes ou que les hommes entre eux. Ici le combat des femmes se situe à l'intérieur du groupe social 'femmes' dans lequel les discours et des propos sont loin de susciter une concession entre elles à cause de conflit d'intérêt souvent commandité par la domination masculine et le syndrome de la reine d'abeille ${ }^{15}$.

Ainsi, en cherchant à conserver leurs fonctions politiques, certaines femmes en exercice de leurs fonctions vont professer leur acte de foi 'politicienne' à chaque moment où l'occasion se présente. Cet acte de foi se traduit par : Je remercie le chef de l'Etat pour la paix retrouvée sans laquelle l'activité conviée n'aurait pas eu lieu ${ }^{16}$. La raison du recours à ce

${ }^{15}$ Le syndrome de la reine d'abeille est la pratique selon laquelle une femme au pouvoir ne veut pas collaborer, mieux s'unir à d'autres femmes en vue de partager son expérience. Elle veut que ça soit toujours elle jusqu'à la fin de sa vie.

${ }^{16}$ Cette déclaration renvoie au Djalelo sous Mobutu où les membres du parti-Etat et du gouvernement devraient chanter et danser à la gloire du président-fondateur, guide, de l'éclaireur en la personne de Mobutu. Cette réalité n'est pas certes une particularité féminine. Presque tous les acteurs politiques au pouvoir sous Joseph Kabila ont mobilisé ou mobilisent le même discours d'acte de bonne foi du Chef de l'Etat dans leurs différents discours et communications. Cette pratique n'est pas favorable à l'exercice réel et effectif du pouvoir par les femmes. Par conséquent, elles reproduisent les mêmes faits politiques observés sous Mobutu. Mais alors, pour nous, l'exercice réel et effectif du pouvoir politique par les femmes devrait-être une opportunité de rupture avec les pratiques anciennes non logiques (où les buts et les moyens ne coïncident pas) pour un changement structurel. Comme l'a fait remarquer 
culte de personnalité se fonde sur la précarité du statut de l'élite comme l'a attesté Vilfredo Pareto. Cette précarité favorise un déplacement de source de légitimité des institutions politiques provinciales du bas (souverain primaire) vers le haut (le chef de l'Etat, le patron).

De ce fait, les femmes détentrices du pouvoir s'emploient plus au clientélisme politique que de lutter en faveur de la consolidation du leadership féminin. Elles sont plongées dans la quête de satisfaction de leur le patron (chef de l'Etat ou le Gouverneur ou encore l'autorité morale du parti) que de chercher à améliorer leur statut politique. Cette attitude traduit l'élitisme de procuration ou de convention en Province Orientale. Ce constant rejoint celui de Gertrude Mainda selon lequel au Zaïre, les femmes sous la deuxième République n'ont pas usé de leurs fonctions politiques et n'avaient pas les moyens de s'en servir pour des engagements sociopolitiques remettant en cause les inégalités entre hommes et femmes (Mianda G., 1995, p. 75).

\section{$2^{\circ}$ Sur les partis politiques}

Les militantes politiques dont les présidentes de ligue des femmes ${ }^{17}$ ont avoué que le manque de financement des activités visant à renforcer les capacités des femmes militantes est une réalité palpable dans nombreux partis politiques, faible visibilité opérationnelle du forum d'appropriation de la résolution 1325, la quête de la facilité chez certaines militantes politiques et faible cohésion entre les femmes. Dans certains cas, les partis politiques constituent un mur de verre en défaveur de la participation politique des femmes. Cette réalité rejoint l'essentiel des démonstrations de Nicole-Claude Mathieu (1991) en prouvant que les différences de sexe sont socialement organisées pour maintenir la minorisation et 1'exclusion des femmes, notamment dans l'espace public en lui niant son statut de sujet social.

Les difficultés d'ordre structurel et interactionniste constituent les « formes matérielles » et les «formes mentales » justifiant les relations de domination, lien entre la matérialité des rapports de pouvoir et la mentalité des individus au sein de la province Orientale. Notre analyse est la continuité de la recherche de Colette Guillaumin (1992). Dans la plupart de partis politiques, l'idéologie promue et l'organisation fonctionnelle sont loin d'aboutir à une réelle émancipation des militantes politiques. La tendance dominante tend à renforcer la domination masculine. Cette domination se

Srilatha Batliwala en ce sens qu'Au final, pour transformer la société, l'empowerment des femmes doit devenir une force politique, c'est-à-dire un mouvement de masse mobilisé qui conteste et transforme les structures de pouvoir existantes pour le bien-être de la nation (Bacqué M. H. \& Biewener C., 2015, p. 33).

${ }^{17}$ La ligue des femmes est un organe chargé de mobiliser les militantes politiques au travers des actions collectives dans le but d'influencer le système politique en faveur du leadership politique féminin. 
repose sur l'exclusion expresse de la femme en faveur de l'homme. Pierre Bourdieu a observé le rapport homme-femme sous une forme de domination masculine. Il déduit que cette domination est tellement ancrée dans les inconscients des hommes et des femmes, membres de cette société au point qu'ils ne l'aperçoivent plus, tellement accordée à leurs attentes. Et qu'ils avaient du mal à la remettre en question (Bourdieu P.,1998, pp. 40-43). La domination masculine a engendré ainsi l'effet Matthieu (Rossiter M.W., 11 | 2003) ${ }^{18}$ en défaveur d'une consolidation du leadership féminin.

Au de-là de ces difficultés, les femmes politiques nourrissent d'ambition permettant de faire preuve de leur capabilité ou non dans le maintien de leur statut. S'agissant de leur ambition en tant que femmes politique, certaines femmes ont émis le vœu de briguer un mandat politique à travers une nomination et une élection ou le deux à la fois. Comme c'est le cas de certains honorables députés provinciaux nommés ministres provinciaux, a signifié Madame Lucie Basonea, Députée Provinciale. Et d'autres l'ont exprimé en ce propos : Elles comptent se maintenir dans la politique afin d'ouvrer pour le changement des mentalités de citoyens et citoyennes et de servir le pays avec abnégation. C'est le cas de madame Niki, ministre provinciale.

\section{Discussion des résultats}

La Province Orientale ne disposait pas d'une politique provinciale Genre, ni le plan provincial de la mise en œuvre de la Résolution $1325 \mathrm{du}$ Conseil de Sécurité des Nations Unies. Même l'Assemblée provinciale de ladite province n'avait voté aucun édit sensible au Genre ${ }^{19}$. Cette réalité traduit la faible volonté politique affichée par les membres du gouvernement provincial et les députés provinciaux dans la mise en œuvre de l'article 3 de l'Objectif de Millénaire pour le développement à savoir promouvoir l'égalité des sexes et l'autonomisation des femmes. Par ailleurs, cette faible volonté politique n'était visible que dans les diverses commémorations de la journée internationale de la femme, les 16 jours d'activiste contre les violences basées sur les femmes et autres activités touchant les femmes.

A travers ces grands moments de commémoration, le Gouverneur de province, le Président de l'Assemblée provincial et le Ministre provincial du Genre s'investissaient en prononçant des discours glosant leur engagement à

\footnotetext{
${ }^{18}$ Effet Matthieu se rapporte à la sur reconnaissance de ceux qui ont le plus (pouvoir politique, pouvoir économique,...). Cette pensée est tirée de l'Evangile de Saint Matthieu : Car à celui qui a il sera donné, et il sera dans la surabondance ; mais à celui qui n'a pas, même ce qu'il a lui sera retiré. (Matthieu13, 12).Pour notre cas, nos structures sociales et politiques tendent à renforcer le pouvoir de l'homme en défaveur de la femme.

${ }^{19}$ Malgré les différents appuis techniques des partenaires techniques et financiers et même les organisations de la société civile pour renforcer les capacités des femmes dans l'élaboration des édits sensibles au Genre.
} 
mettre fin aux discriminations dont sont victimes les femmes. Leur engagement était également plus motivé lorsque ces activités en faveur des femmes étaient financées par les bailleurs de fonds.

Malgré cela, les changements tant souhaités tardaient à se faire voir dans la mesure où le Conseil Provincial de la Femme (un organe technique du gouvernement) n'existait que sur papier ${ }^{20}$, la faible performance de prestation du ministère provincial du genre et de la division provinciale du genre, famille et enfant comme l'a démontré le rapport d'audit Genre du ministère réalisé sous l'appui du Programme de Nations Unies pour le Développement, PNUD en sigle ${ }^{21}$. Cette faible performance s'expliquait en partie par le fait que ces institutions avaient une faible connaissance sur les notions de Genre et que le budget provincial de la Province Orientale n'était pas sensible au genre. Dans ce contexte, il était difficile d'instaurer une vision commune de la promotion du genre dans cette province.

Face à ce constat, il y a lieu de s'interroger sur les attitudes des femmes leaders politiques dans la consolidation du leadership féminin et la promotion de l'égalité entre les sexes. Nous comprenons que les femmes leaders politiques mobilisaient les femmes de masses et celles de la société civile pour constituer un poids politique en vue de se hisser au pouvoir mais une fois au pouvoir, la plupart de ces femmes rompaient la communication avec les différentes catégories des femmes, c'était le cas avec les députés élues venues de certains territoire. Elles optent délibérément le choix de s'allier à « la stabilité/confort » qu'offre le système néo patriarcal ${ }^{22}$. «Ekomi tango na yo, yo mpe liya ya yo ${ }^{23} \gg$. Elles ne s'employaient plus à travailler pour le changement structurel convenu et promis en faveur de la promotion des droits des femmes mais en la réalité, elles optaient pour la résignation face à la

${ }^{20}$ Cet organe est présidé par le gouverneur de la Province et les actrices de la société civile œuvrant pour la promotion des droits de femmes en font partie. Il faut également souligner que pendant le règne du Gouverneur Bamanisa, Il y avait une conseillère en Genre au Gouvernorat de Province Orientale.

${ }^{21}$ Signalons que ce rapport d'audit genre de 2012 était une de recommandations du Rapport National Genre de la RDC produit en 2011 qui voulait mettre en évidences les causes principales des inégalités fondées sur les sexes en défaveur des femmes et des filles congolaises. Le Rapport National Genre a révélé entre autres que la situation des femmes et des filles congolaises reste très préoccupante et les inégalités fondées sur les sexes très persistantes dans tous les secteurs de la vie nationale (civil, politique, économique, social, culturel...) faute d'une prise en compte suffisante de la dimension Genre comme transversale et stratégique dans les politiques, programmes et projets du développement national, et ce, malgré la production et la mise en œuvre depuis 2004 d'une Stratégie Nationale d'Intégration du Genre dans les politiques et programmes du Développement National.

${ }^{22}$ Ce type de système offre l'opportunité de se positionner politiquement mais sans les donner le pouvoir d" agir et de décider librement.

${ }^{23}$ Ce propos se traduit littéralement en ce sens : l'heure est venue pour la femme leader de s'enrichir de son pouvoir. 
démocratie masculine. Elles se noyaient dans les lamentations interminables, elles s'inclinaient devant le rouleau compresseur, de toute façon elles n'y pouvaient rien puis qu'elles étaient corrompu par le pouvoir à cause des dividendes matérielles, financières ou symboliques reçues. Elles étaient incapables de contribuer à mettre en place un mécanisme garantissant la pleine participation des femmes au même titre que les hommes. En fait, certaines femmes leaders n'avaient pas de mot à dire à face leur supérieur, leur parrain politique, leur autorité morale du parti politique dans l'exercice de ses fonctions. Elles n'avaient pas assez d'argument pour convaincre la majorité des collègues à adhérer à leur vision en brisant le plafond de verre stéréotypé. Elles ont préféré se résigner face à l'usurpation et empiétement du pouvoir de leur chef. Certaines femmes politiques ont allé plus loin en interpellant les autres femmes politiques et celles de la société civile à abandonner des actions de revendications qui risquaient de compromettre les fonctions exercées par leur parrain politique, leur chef, pour la seule raison que les hommes politiques sont nos enfants, leur papa, nous avons l'obligation de les protéger! Cette attitude de certaines femmes montrent qu'elles sont des femmes leaders de procuration et fabriquées pour redorer carte genre de la Province Orientale.

Les administrés ne plaignaient de l'absence de l'autorité communale et de style de son leadership en ces termes : "Actuellement, sollicitée une audience auprès de l'autorité et recevoir une suite favorable est un problème pour l'administration communale de Kabondo. La plupart de temps, l'autorité municipale est toujours absente de son bureau. C'est le Bourgmestre adjoint qui traite très souvent les dossiers mais il n'a pas le pouvoir de décider sans aval de son chef direct ». La Bourgmestre exerçait sa fonction par procuration. Malgré ces plaintes, aucune décision n'avait été prise pour l'amener à appliquer le management de responsabilité dans la gestion de son entité. Elle est maintenue car elle est femme et membre du PPRD, parti au pouvoir ${ }^{24}$. Cette attitude de résignation a fait perdre aux femmes leur opportunité de consolider le leadership féminin et d'améliorer la gouvernance politique comme le suggère l'article 3 de l'Objectif de Millénaire pour le développement. Ce rendez-vous manqué pour les femmes leaders politiques a fait qu'elles soient qualifiées de convention. Car elles n'étaient pas à la hauteur de la performance d'attendue et qu'elles n'ont pas pu démissionner pour leur incapacité en tant femmes, à interagir avec leurs clients.

L'examen de propos recueillis auprès de femmes nous pousse à croire que les femmes leaders politiques ont le souci d'exercer, le plus longtemps possible, leur mandat politique, de maintenir le cap en politique. Ainsi, ces

${ }^{24}$ Il y a lieu d'épingler également le ministre du Genre, malgré sa faible performance en matière de la promotion du Genre, elle avait maintenu sa fonction jusqu'à l'investiture du nouveau gouvernement provincial conduit par Jean Bamanisa Saidi. Madame Angele Uvon jouissait du privilège découlant du pouvoir discrétionnaire du Gouverneur de Province. 
femmes tout comme leurs collègues masculins sont prêts à user de tous les moyens pour y arriver, même la transhumance politique. En politique, selon eux, il n'y a pas de cadeau, mais de compromis et de compensation. Ainsi, nous sommes dans un système de dons et contre-dons, comme l'a théorisé Marcel Mauss. Il a proposé une première théorie de l'échange en mettant en évidence dans la communauté primitive des "systèmes de prestations sociales » gouvernés par les trois obligations régissant le «don»: donner, recevoir et rendre (Mauss M., 1993). Cette trilogie peut également s'observer dans la conquête d'un mandat politique à divers niveaux par le bas (élection) et par le haut (nomination). Autrement dit, le don est un instrument de domination et de séduction d'après Alain Testart (Journet N., 2016, p. 59).

Par ailleurs, la sociologie électorale de la RDC laisse voir que la logique communautaire dans l'élection des candidats n'est pas toujours un acquis. Ainsi, les Congolais ont été élus en dehors de leur milieu d'origine ethnique. C'est le cas notamment de Madame Eve Bazaiba qui, en janvier 2007, était élue Sénatrice sur la liste MLC pour la circonscription de Kinshasa.

La présence des députés femmes musulmanes comme mesdames Aisha Kwanza (député provinciale de la circonscription de Bambesa) et Eve Bazaiba (député nationale de la circonscription de Basoko) contredit l'opinion publique sur la passivité des femmes musulmanes dans la jouissance de leurs droits politiques. Cette passivité serait d'après cette opinion conditionnée par la socialisation des valeurs islamiques. Certaines valeurs seraient donc en désaccord avec le militantisme politique. Cette hypothèse est balayées par la pensée de Maxime Rodinson dans l'islam et le capitalisme (Rodinson M., 1966), les valeurs musulmanes ne sont pas contradictoires avec l'activité économique et ne conduisent pas au fatalisme et à la passivité. A notre avis, cette passivité pourrait se justifier par la sous information sur l'exercice des droits et libertés fondamentales.

Au regard des ambitions affichées et des difficultés d'ordre structurel et interactionniste, certaines femmes leaders politiques grâce à leur compétence, leur savoir-faire et faire-être ont conservé leur statut d'agent de changement structurel et interactionniste dans une perspective féministe justifiant la qualification des élites féminines de conviction ${ }^{25}$.

\footnotetext{
${ }^{25}$ A ce sujet, le récit de Madame Marie Bhamoko Deuda à la territoriale demeure très éloquent. Cette dame a une expérience de l'administration territoriale très riche, mais sous exploitée en ce sens qu'elle est détentrice d'une licence en Sciences Politiques et Administratives de l'Université de Kisangani en 1980 et que la plupart des agents de la territoriale reconnaissent son savoir- faire (compétence) dans la gestion des entités administratives (territoire ou municipalité) pour avoir été Administrateur du territoire assistant d'Isangi, Banalia sous Mobutu. Mais aucune fois, elle n'a été promue au poste titulaire de la territoriale alors que d'autres avec moins d'expérience sont promues titulaires. Elle est obligée de travailler parfois avec des néophytes avec une faible capacité d'écoute. Son récit traduit le rapport hommefemme en termes de la sous-exploitation de la compétence féminine au sein de
} 
Mais hélas, cette catégorie est minoritaire raison pour laquelle leur impact sur la gestion reste très faible. Ce qui rejoint les conclusions de Chandan Kumar Jha et sudipta Sarang (Chandan Kumar Jha \& Sudipta Sarang, 2018) sur la participation des femmes au pouvoir politique. D'après ces auteurs, Autant que la participation des femmes au pouvoir politique s'inscrit dans le temps (la durée) et dans la structuration (organisation), autant le déclin de la corruption est important. Le lien de causalité augmente donc avec le temps. Même lorsque les femmes politiques sont mieux en réseaux, la corruption continue d'être réprimée par leur présence. Un autre facteur est que les femmes fixent des priorités politiques différentes de celles des hommes. Elles sont plus susceptibles de s'engager dans des domaines où il y a moins de possibilités d'abus, comme les soins de santé, l'éducation et la lutte contre la pauvreté. Chandan Khumar Jha suggère pour le cas de la RDC, d'attribuer la moitié des sièges du parlement et des postes ministériels aux femmes et de leur donner réellement l'occasion d'utiliser leur capabilité (pouvoir). Au final, il y aura rapidement les résultats. Avant la fin de la législature, à condition qu'il n'y ait pas d'autres bouleversements majeurs, la corruption aura très probablement diminué. Cette règle s'applique aujourd'hui quasiment partout au monde.

En observant la situation des institutions provinciales de la Province Orientale, les statistiques font état de 96 députés dont huit femmes (assemblée provinciale) et douze membres du gouvernement provincial dont deux femmes. À ce stade, les effets de la participation des femmes leaders politique sur la corruption et le bien être est loin d'être une réalité.

La majorité des femmes se rabattent des aménagements parfois en leur défaveur se traduisant souvent par les non-dits, la peur face aux réactions des autorités politiques, le manque de compétence dans certaines matières. Cette situation engendre une pérennisation anti féministe d'où la qualification des élites féminines de convention. Ces femmes politiques sont incapables de renoncer aux modes rusés de leur émergence de peur qu'elles ne perdent leur statut. Femmes leaders politiques de convention (personnalité inféodée) et celles de conviction (personnalité affirmée) sont les deux types de personnalités constituant les fruits de la mise en œuvre de la capabilité des élites féminines dans l'exercice de leur militantisme, fonction et mandat

l'administration. Malgré ces faits discriminatoires, elle demeure constante dans sa façon d'être, de faire et d'agir. Ainsi, elle a affirmé qu'elle demeure dans sa situation de sous valorisation pourvu que sa conscience soit tranquille et qu'elle soit un modèle d'engagement féminin et de personnalité pour d'autres femmes aspirantes. En qualité de Bourgmestre Adjoint de Mangobo puis de Lubunga, elle se contente juste d'une reconnaissance symbolique. Entretien réalisé avec la Bourgmestre adjointe de Lubunga, Madame Marie Bhamoko Deuda, le 08 octobre 2014 de $10 \mathrm{~h}$ à $12 \mathrm{~h} 25^{\prime}$. 
politique. La catégorie majoritaire étant celle regroupant les femmes leaders de convention, cette situation montre comment la transformation du système n'est qu'artificielle et non fondamentale. Ainsi, le sous-système est passée du patriarcal au néo-patriarcal en adoptant des discours et des actions sensible au genre sans réellement apportées une profonde transformation en matière de rapports sociaux de sexes.

\section{Conclusion}

L'émergence des femmes leaders politiques s'inscrit dans un champ de la subversion des normes socialement établies, de digression du déterminisme social. Le fait pour ce groupe des femmes leaders politiques d'échapper tant soit peu au déterminisme social a engendré des identités subversives comme nous l'avons observé dans un article sur les images contrastées des femmes de la société civile (Salumu Laumu Omeyaka, 2017).

Partant d'expériences diverses, ces femmes sont, de manières conscientes ou inconscientes, victimes de la socialisation de la division sexuée de l'espace et celle du travail dans leur environnement social. Cette socialisation est le moteur de difficultés d'ordre structurel et interactionniste que rencontrent les femmes dans l'exercice de leur pouvoir politique en Province Orientale. Leur ambition constitue pour certaines, un moyen de résistance au jeu performatif du genre (subversion) et pour d'autre, un moyen de maintien du jeu performatif (performance). Dans la mesure où certaines d'entre-elles ont transcendé les barrières politiques et sociales érigées contre elles en usant de leur capabilité en terme de connaissance, savoir -faire, carnet d'adresse, liens sociaux en vue de leur permettre d'acquérir une place dans les institutions politiques en Province Orientale et d'autres par des simples procurations ou recommandations sans compétence.

L'ensemble de difficultés rencontrées par les femmes ont impacté négativement sur la double lutte des femmes leaders en Province Orientale :

La lutte par le bas: Il s'agit d'une lutte des femmes visant à instaurer et consolider la cohésion entre les femmes en vue de combattre les inégalités sociales, économiques et politiques observées en défaveur de la femme. Cette lutte était observable à travers certaines structures regroupant les femmes à savoir le collectif de femmes de la Province Orientale, le cadre de concertation permanent de la femme congolaise qui sont des plateformes des réseaux des femmes de la société civile et/ou des organisations politiques. Mais le constat est que les femmes politiques n'entretiennent pas de collaboration permanente et régulière avec leurs consœurs de la société civile. Par contre, nous avons observé une collaboration parfois controversée à cause des intérêts politiciens. Ce rapport mitigé entre les femmes leaders de ces deux sphères a impacté négativement la consolidation du leadership féminin dans de la province Orientale. 
La lutte par le haut: Il s'agit d'une lutte au sein des institutions politiques provinciales où les femmes détentrices du pouvoir devraient influer la conduite des institutions en faveur de la prise en compte de leurs désidératas en termes de politiques publiques sensibles au genre. Les intérêts politiciens ont primé sur la promotion du leadership féminin en province Orientale en ce sens qu'il y a eu absence des Politiques publiques formelles touchant les besoins stratégiques des hommes et de femmes telle que la Provinciale Genre en Province Orientale ou encore le plan provincial de la mise en œuvre de la Résolution 1325 des Nations Unies. A cela s'ajoute l'absence de certaines structures d'accompagnement de la femme à savoir le Conseil Provincial de la femme. Une telle structure, en sa qualité d'organe technique, devrait réfléchir et proposer au gouvernement provincial voire central des alternatives possibles pour améliorer la participation politique des femmes au regard des données fournies par la sociologie électorale de la dite province. Mais cet organe se réduit aux aspects festifs tels que la commémoration du 08 mars. Puisqu'il serait constitué par les femmes leaders de convention et non de conviction, au lieu d'organiser de table ronde sur de question touchant le leadership féminin, par exemple le mode d'alignement des candidats et candidates sur la liste électorale appelé "La liste zébrée-bloquée », ce qui revient à dire "l'alternance homme-femme, femme-homme » sur les listes des candidatures à soumettre aux suffrages, tout en permettant de prendre en compte les groupes sociaux défavorisés. L'adoption de ce mode donnerait la chance aux femmes de se faire élire comme l'ont fait observer Hailey Hayeon et Jungmin Lee dans leur analyse sur l'électorat féminin. Selon eux, L'effet d'exposition obligatoire sur le vote représente un mécanisme grâce auquel des politiques d'action positive telles que les quotas peuvent améliorer les perspectives des candidats des groupes sous-représentés (Hailey Hayeon Joo \& Jungmin Lee, 2018).

\section{References:}

1. Alami Mchichi H. (2002). Genre et politique. Les enjeux de l'égalité hommes-femmes entre islamisme et modernisme, Paris, L'Harmattan .

2. Bacqué M. H. \& Biewener C. (2015). L'empowerment, une pratique émancipatrice. Paris, La Découverte.

3. Bailey, F. G. (1988). Humbuggery and manipulation: the art of leadership, Ithaca, Cornell University Press.

4. Bardin L. (2016). L'analyse de contenu, Paris, PUF.

5. Bourdieu P. (1980). Le sens pratique, Paris, Minuit.

6. Bourdieu P. (1998). Domination masculine, Paris, Seuil.

7. Butler J. (2005). Trouble dans le genre. Pour un féminisme de la subversion, Paris, La Découverte.

8. Butler J., .. (2006). Défaire le genre, trad., Paris, Editons Amsterdam. 
9. Chandan Kumar Jha \& Sudipta Sarang. (2018, July). Women and corruption: What positions must they hold to make a difference? Journal of Economic Behavior \& Organization, 151, 219-233. Récupéré sur https://doi.org/10.1016/j.jebo.2018.03.021

10. Foucault M. (1976). Histoire de la sexualité I : la volonté de savoir, Paris, Gallimard.

11. Gaxie D. (1979). le sens caché, Paris, Ed. Le Seuil. Consulté le 01 Mai 2016, sur http://www.persee.fr/docAsPDF/reso_07517971_1987_num_5_22_1237.pdf

12. Grawitz M. et Leca J. (1979). Traité de science politique,Paris, PUF.

13. Guillaumin C. (1992). Sexe, Race et Pratique du pouvoir, Paris, côtéfemmes.

14. Hailey Hayeon Joo \& Jungmin Lee. (2018). Encountering female politicians. Journal of Economic Behavior \& Organization, 151, 88122. Récupéré sur https://doi.org/10.1016/j.jebo.2018.05.004

15. Heim P. \& Susan Murphy S. (2004). La femme est un loup pour la femme : Comprendre et résoudre les conflits entre femmes, Paris, Payot.

16. Héritier F. (1996). Masculin-féminin I. la pensée de la différence, Paris, Odile Jacob.

17. Hirschman A. (1982). Shifting Involvments: Private Interest and Public Action. (Fayard,1995, Éd.) Princeton, NJ, Princeton University Press.

18. Journet N. (2016). Les grands penseurs des sciences humaines, Auxerre, éd. Sciences sociales.

19. Mathieu N.-C. (1991). L'anatomie politique. Catégorisations et idéologies du sexe, Paris, Côtes-femmes.

20. Mauss M. (1993). Sociologie et Anthropologie, Paris, PUF.

21. Mianda G. (1995). «Dans l’ombre de Ia « démocratie » au Zaïre : la remise en question de I'émancipation mobutiste de la femme. Mianda G., "Dans l'ombre de Ia " démocratie » au Zä̈re : la remise eRevue canadieme des études africaines, no 29.

22. Ministere du Genre de la Famille et de l'Enfant, R. (2012). Rapport d'audit national en genre des institutions publiques et privées de la $R D C$, Kinshasa.

23. Odimba C., N. J.-R. (2012). Participation des femmes dans les processus de la paix et la prise de décision Politique en République Démocratique du Congo. Rapport, Alert international.

24. Omasombo J. (2009). RDC Biographies des acteurs de la Troisième République. Kinshasa, Lubumbashi, Tervuren, CEP-CERDACMRAC. 
25. Omasombo J. et Kennes E. (2006.). RDC.Biographies des acteurs de la Transition (Juin 2003-Juin 2006). Kinshasa, Lubumbashi, Tervuren, CEP-CERDAC-MRAC.

26. Rodinson M. (1966). Islam et capitalisme,. Paris, Éd. du Seuil.

27. Rossiter M.W. (11 | 2003, février 2010 16). L'effet Mathilda en sciences. Les cahiers du CEDREF [Enligne]. Consulté le 21 décembre 2016, http://cedref.revues.org/503

28. Salumu Laumu Omeyaka, B. (2017, August). Les Images Contrastées des Femmes Émergentes De La Société Civile et Transformation des Rôles Sociaux Sexués en Province Orientale Post-Mobutu. European Scientific Journal,

13. https://eujournal.org/index.php/esj/issue/view/289 DOI: HYPERLINK "http://dx.doi.org/10.19044/esj.2017.v13n23p129" It " "blank" 10.19044/esj.2017.v13n23p129

29. Sow, F. (2009). La recherche féministe francophone. Langue, identités et enjeux, Paris, Karthala.

30. WITTIG M. (2001). La pensée straight, Paris, Ed. Balland. 